JOURNAL OF OPTIMIZATION, DIFFERENTIAL EQUATIONS AND THEIR APPLICATIONS (JODEA) Volume 26, Issue 2, December 2018, pp. 37-54, DOI 10.15421/141809

ISSN (print) 2617-0108

ISSN (on-line) $\mathrm{xxxx}-\mathrm{xxxx}$

\title{
OPTIMAL CONTROL PROBLEM FOR SOME DEGENERATE VARIATION INEQUALITY: ATTAINABILITY PROBLEM
}

\author{
Nina V. Kasimova*
}

\begin{abstract}
We study an optimal control problem for degenerate elliptic variation inequality with degenerate weight function of potential type in the so-called class of $H$-admissible solutions. Using an appropriate regular algorithm of perturbation, we prove attainability of $H$-optimal pairs via optimal solutions of some non-degenerate perturbed optimal control problems.
\end{abstract}

Key words: optimal control problem, elliptic variation inequality, degenerate weight function of potential type, $H$-admissible solution, $H$-optimal solution, perturbation.

2010 Mathematics Subject Classification: 49J20, 49K20, 58J37, 35J50.

Communicated by Prof. O.P. Kupenko

\section{Introduction}

The aim of this paper is to study optimal control problems associated to degenerate elliptic variational inequalities in the so-called class of $H$-admissible solutions. Dealing with degenerate problems leads us to the concept of weighted Sobolev spaces such as $W(\Omega, \rho d x)$ (see for example [5]), where $\rho$ is degenerate (in some sense) weight function, such that the differential operator associated to our problem is not coercive in the classical sense. Hence, the classical approach to investigate mentioned problems can't be used. In [17] was proposed an alternative method for solving optimal control problems for degenerate variational elliptic inequality, using Hardy-Poincare inequality.

It is known that smooth functions are, in general, not dense in the space $W(\Omega, \rho d x)$ that leads to the issues related to non-uniqueness of the setting of correspondent boundary value problem and as a consequence, to several possible settings of an optimal control problem associated to the mentioned control object. If we consider the space $H(\Omega, \rho d x)$ which is the closure of $C_{0}^{\infty}(\Omega)$ in $W(\Omega, \rho d x)$, then $H(\Omega, \rho d x) \neq W(\Omega, \rho d x)$, in general (see, for example [15]). In literature this fact is called the Lavrentiev phenomenon.

In applications a degenerate weight function $\rho$ appears as the limit of the sequence of non-degenerate weights $\rho^{\varepsilon}$, for which the corresponding "approximate" problem is solvable. In this paper we interested in attainability of $H$-optimal solutions to degenerated problems via optimal solutions of non-degenerated problems, namely, we show that each optimal solution to the degenerate problem can

\footnotetext{
* Department of Integral and Differential Equations, Taras Shevchenko National University of Kyiv, 64/13, Volodymyrska Street, Kyiv, Ukraine, 01601, zadoianchuk.nv@gmail.com

(c) N. V. Kasimova, 2018.
} 
be attained by admissible solutions to perturbed problems, however there exists at least one optimal solution of degenerated problem which can be attained by optimal solutions to appropriate perturbed problems.

\section{Notations and preliminaries}

Let $\Omega \subset \mathbb{R}^{N}(N \geq 3)$ be an open bounded set with regular boundary $\partial \Omega$ such, that $0 \in \mathbb{R}^{N}$ is an inner point of $\Omega$. Hereafter we will denote a locally convex space of all infinitely differentiable functions with supports in $\Omega$ by $C_{0}^{\infty}(\Omega)$.

Let $\rho: \Omega \rightarrow \mathbb{R}$ be a given function such that: $\rho(x)>0$ a.e. on $\Omega$,

$$
\rho \in L^{1}(\Omega), \rho^{-1} \in L^{1}(\Omega), \nabla \ln \rho \in L^{2}\left(\Omega ; \mathbb{R}^{N}\right) \text { i } \rho+\rho^{-1} \notin L^{\infty}(\Omega) .
$$

Hereafter, we assume that there exists a closed subset $\mathcal{O}$ of the set $\Omega$ such that

$$
\operatorname{dist}(\mathcal{O}, \partial \Omega)=\varepsilon, \quad \rho>\varepsilon \text { м.с. в } \Omega \backslash \mathcal{O}, \quad \text { і } \quad \rho \in L^{\infty}(\Omega \backslash \mathcal{O})
$$

for some $\varepsilon>0$. In other words we assume that conditions (2.1) are not typical for boundary layer of the set $\Omega$.

Weighted spaces. We call a nonnegative function $\rho$ with properties $(2.1)-(2.2)$ degenerate and consider weighted Hilbert spaces $L^{2}(\Omega, \rho d x)$ and $L^{2}\left(\Omega, \rho^{-1} d x\right)$, saying that

$$
f \in L^{2}(\Omega, \rho d x) \text { if }\|f\|_{L^{2}(\Omega, \rho d x)}^{2}=\int_{\Omega} f^{2} \rho d x<+\infty,
$$

and $g \in L^{2}\left(\Omega, \rho^{-1} d x\right)$ if $\|g\|_{L^{2}\left(\Omega, \rho^{-1} d x\right)}^{2}=\int_{\Omega} g^{2} \rho^{-1} d x<+\infty$.

We define the space $W=W(\Omega, \rho d x)$ as a set of functions $y \in W_{0}^{1,1}$ for which the norm

$$
\|y\|_{\rho}:=\left(\int_{\Omega} y^{2} \rho d x+\int_{\Omega}|\nabla y|_{\mathbb{R}^{N}}^{2} \rho d x\right)^{1 / 2}
$$

is finite, and the space $H=H(\Omega, \rho d x)$ as the closure of the space $C_{0}^{\infty}(\Omega)$ with respect to the norm (2.3).

Note, that spaces $W$ and $H$ are reflexive Banach spaces with respect to the norm (2.3) due to the estimate

$$
\int_{\Omega}|\nabla y| d x \leq\left(\int_{\Omega} \rho|\nabla y|_{2}^{2} d x\right)^{1 / 2}\left(\int_{\Omega} \rho^{-1} d x\right)^{1 / 2} \leq C\|y\|_{\rho},
$$

where $|\eta|_{2}=\left(\sum_{k=1}^{N}\left|\eta_{k}\right|^{2}\right)^{1 / 2}$.

Since the smooth functions are in general not dense in the weighted Sobolev space $W$, it follows that $H \neq W$; that is for a "typical" degenerate weight $\rho$ the identity $W=H$ is not always valid (for corresponding examples we refer to 
$[1,12,13])$. However, if $\rho$ is a non-degenerate weight function, that is, $\rho$ is bounded between two positive constants, then it is easy to verify that $W=H=H_{0}^{1}(\Omega)$. We recall that the dual space of $H$ is $H^{*}=W^{-1,2}\left(\Omega, \rho^{-1} d x\right)$ (for more details see [5]).

Remark 2.1. [16, Remark 1] In the case when the weight $\rho^{-1} \in L^{1}(\Omega)$, the space $H(\Omega, \rho d x)$ is continuously embedded into the space $W_{0}^{1,1}(\Omega)$.

Let us consider the next concept [17]

Definition 2.1. We say $\rho: \Omega \rightarrow \mathbb{R}$ is the weight function of potential type if $\rho$ satisfies conditions $(2.1)-(2.2)$ and there exists such constant $\widehat{C}(\Omega)>0$, that the following inequality is fulfilled:

$$
-\widehat{C}(\Omega) \leq-\triangle \ln \rho(x)-\frac{1}{2}|\nabla \ln \rho|_{\mathbb{R}^{N}}^{2}<\frac{2 \lambda_{*}}{|x|_{\mathbb{R}^{N}}^{2}}=\frac{(N-2)^{2}}{2|x|_{\mathbb{R}^{N}}^{2}} \quad \text { in } \Omega .
$$

In this case the function $V(x)=-\triangle \ln \rho(x)-\frac{1}{2}|\nabla \ln \rho|_{\mathbb{R}^{N}}^{2}$ is called Hardy potential for the weighted function $\rho$.

\section{Elliptic Variational Inequalities.}

Let $V$ be a Banach space and $K \subset V$ be a closed convex subset. Suppose also that $A: K \rightarrow V^{*}$ is a nonlinear operator and $f \in V^{*}$ is a given element of the dual space.

Let us consider the following variational problem: to find an element $y \in K$ such that

$$
\langle A y, v-y\rangle_{V} \geq\langle f, v-y\rangle_{V}, \quad \forall v \in K
$$

Referring to [9], we make use of the following assumptions.

Hypothesis 1. There exists a reflexive Banach space $X$ such that $X \subset V^{*}$, the imbedding $X \hookrightarrow V^{*}$ is continuous, and $X$ is dense in $V^{*}$.

Hypothesis 2. There can be found a duality mapping $J: X \rightarrow X^{*}$ such that $\forall y \in K, \forall \varepsilon>0$ there exists an $y_{\varepsilon} \in K$ such that $A\left(y_{\varepsilon}\right) \in X$ and

$$
y_{\varepsilon}+\varepsilon J\left(A\left(y_{\varepsilon}\right)\right)=y \text {. }
$$

Theorem 2.1. [9, Theorem 8.7] Assume that Hypothesis 1 and Hypothesis 2 hold true. Let operator $A: V \rightarrow V^{*}$ be monotone, semicontinuous, bounded and satisfy the following assumption: there exist an element $v_{0} \in K$ such that

$$
\frac{\left\langle A y, y-v_{0}\right\rangle_{V}}{\|y\|_{V}} \rightarrow+\infty \quad \text { as } \quad\|y\|_{V} \rightarrow \infty, y \in K .
$$

Then for any solution $y$ of variational inequality (2.5) the inclusion Ay $\in X$ takes plase provided $f \in X$.

Smoothing. Throughout the paper $\varepsilon$ denotes a small parameter which varies within a strictly decreasing sequence of positive numbers converging to 0 . When we write $\varepsilon>0$, we consider only the elements of this sequence, while writing $\varepsilon \geq 0$, we also consider its limit $\varepsilon=0$. 
Definition 2.2. We say that a weight function $\rho$ with properties $(2.1)-(2.2)$ is approximated by non-degenerated weight functions $\left\{\rho^{\varepsilon}\right\}_{\varepsilon>0}$ on $\Omega$ if:

$$
\begin{gathered}
\rho^{\varepsilon}(x)>0 \text { a.e. in } \Omega, \rho^{\varepsilon},\left(\rho^{\varepsilon}\right)^{-1} \in L^{\infty}(\Omega), \forall \varepsilon>0, \\
\rho^{\varepsilon} \rightarrow \rho,\left(\rho^{\varepsilon}\right)^{-1} \rightarrow \rho^{-1} \text { in } L^{1}(\Omega) \text { as } \varepsilon \rightarrow 0 .
\end{gathered}
$$

Remark 2.2. The family $\left\{\rho^{\varepsilon}\right\}_{\varepsilon>0}$ satisfying properties (2.6)-(2.7) is called the non-degenerate perturbation of the weight function $\rho$.

Examples of such perturbations can be constructed using the classical smoothing. For instance, let $Q$ be some positive compactly supported function such that $L^{\infty} \mathbb{R}^{N}, \int_{\mathbb{R}^{N}} Q(x) d x=1$, and $Q(x)=q(-x)$. Then, for a given weight function $\rho \in L_{l o c}^{1}\left(\mathbb{R}^{N}\right)$, we can take $\rho^{\varepsilon}=(\rho)_{\varepsilon}$, where

$$
(\rho)_{\varepsilon}(x)=\frac{1}{\varepsilon^{N}} \int_{\mathbb{R}^{N}} Q\left(\frac{x-z}{\varepsilon}\right) \rho(z) d z=\int_{\mathbb{R}^{N}} Q(z) \rho(x+\varepsilon z) d z .
$$

In this case we say that the perturbation $\left\{\rho^{\varepsilon}=(\rho)_{\varepsilon}\right\}_{\varepsilon>0}$ of the original degenerate weight function $\rho$ is conctructed by the "direct" smoothing scheme.

Lemma 2.1. [10] If $\rho, \rho^{-1} \in L_{\text {loc }}^{1}\left(\mathbb{R}^{N}\right)$ then the "direct" smoothing $\left\{\rho^{\varepsilon}=(\rho)_{\varepsilon}\right\}_{\varepsilon>0}$ possesses properties (2.6)-(2.7).

Weak compactness criterion in $L^{1}(\Omega)$. Throughout the paper we will often use the concepts of weak and strong convergence in $L^{1}(\Omega)$. Let $\left\{a_{\varepsilon}\right\}_{\varepsilon>0}$ be a bounded sequence in $L^{1}(\Omega)$. We recall that $\left\{a_{\varepsilon}\right\}_{\varepsilon>0}$ is called equi-integrable if for any $\delta>0$ there exists $\tau=\tau(\delta)$ such that $\int_{S}\left|a_{\varepsilon}\right| d x<\delta$ for every $\varepsilon>0$ and every measurable subset $S \subset \Omega$ of Lebesgue measure $|S|<\tau$. Then the following assertions are equivalent:

(i) A sequence $\left\{a_{\varepsilon}\right\}_{\varepsilon>0}$ is weakly compact in $L^{1}(\Omega)$.

(ii) The sequence $\left\{a_{\varepsilon}\right\}_{\varepsilon>0}$ is equi-integrable.

(iii) Given $\delta>0$ there exists $\lambda=\lambda(\delta)$ such that $\sup _{\varepsilon>0} \int_{\left\{\left|a_{\varepsilon}\right|>\delta\right\}}\left|a_{\varepsilon}\right| d x<\delta$.

Theorem 2.2. (Lebesgue's Theorem). If a bounded sequence $\left\{a_{\varepsilon}\right\}_{\varepsilon>0} \subset L^{1}(\Omega)$ is equi-integrable and $a_{\varepsilon} \rightarrow a$ almost everywhere on $\Omega$, then $a_{\varepsilon} \rightarrow a$ in $L^{1}(\Omega)$.

Radon measures and convergence in variable spaces. By a nonnegative Radon measure on $\Omega$ we mean a nonnegative Borel measure which is finite on every compact subset of $\Omega$. The space of all nonnegative Radon measures on $\Omega$ will be denoted by $\mathcal{M}_{+}(\Omega)$. If $\mu$ is a nonnegative Radon measure on $\Omega$, we will use 
$L^{r}(\Omega, d \mu), 1 \leq r \leq \infty$, to denote the usual Lebesgue space with respect to the measure $\mu$ with the corresponding norm

$$
\|f\|_{L^{r}(\Omega, d \mu)}=\left(\int_{\Omega}|f(x)|^{r} d \mu\right)^{1 / r} .
$$

Let $\left\{\mu_{\varepsilon}\right\}_{\varepsilon>0}, \mu$ be Radon measures such that $\mu_{\varepsilon}$ is $*$-weakly convergent to $\mu$ in $\mathcal{M}_{+}(\Omega)$; that is,

$$
\lim _{\varepsilon \rightarrow 0} \int_{\Omega} \varphi d \mu_{\varepsilon}=\int_{\Omega} \varphi d \mu \quad \forall \varphi \in C_{0}\left(\mathbb{R}^{N}\right),
$$

where $C_{0}\left(\mathbb{R}^{N}\right)$ is the space of all compactly supported continuous functions. A typical example of such measures is $d \mu_{\varepsilon}=\rho^{\varepsilon}(x) d x, d \mu=\rho(x) d x$, where $0 \leq \rho^{\varepsilon} \rightarrow$ $\rho$ in $L^{1}(\Omega)$. Let us recall the definition and main properties of convergence in the variable $L^{2}$-space [13].

1. A sequence $\left\{v_{\varepsilon} \in L^{2}\left(\Omega, d \mu_{\varepsilon}\right)\right\}$ is called bounded if

$$
\limsup _{\varepsilon \rightarrow 0} \int_{\Omega}\left|v_{\varepsilon}\right|^{2} d \mu_{\varepsilon}<+\infty
$$

2. A bounded sequence $\left\{v_{\varepsilon} \in L^{2}\left(\Omega, d \mu_{\varepsilon}\right)\right\}$ converges weakly to $v \in L^{2}(\Omega, d \mu)$ if

$$
\lim _{\varepsilon \rightarrow 0} \int_{\Omega} v_{\varepsilon} \varphi d \mu_{\varepsilon}=\int_{\Omega} v \varphi d \mu
$$

for any $\varphi \in C_{0}^{\infty}(\Omega)$ and we write $v_{\varepsilon} \rightarrow v$ in $L^{2}\left(\Omega, d \mu_{\varepsilon}\right)$.

3. The strong convergence $v_{\varepsilon} \rightarrow v$ in $L^{2}\left(\Omega, d \mu_{\varepsilon}\right)$ means that $v \in L^{2}(\Omega, d \mu)$ and

$$
\lim _{\varepsilon \rightarrow 0} \int_{\Omega} v_{\varepsilon} z_{\varepsilon} d \mu_{\varepsilon}=\int_{\Omega} v z d \mu \text { as } z_{\varepsilon} \rightarrow z \text { in } L^{2}\left(\Omega, d \mu_{\varepsilon}\right) .
$$

The following convergence properties in variable spaces hold:

(a) Compactness criterium: if a sequence is bounded in $L^{2}\left(\Omega, d \mu_{\varepsilon}\right)$, then this sequence is compact with respect to the weak convergence.

(b) Property of lower semicontinuity: if $v_{\varepsilon} \rightarrow v$ in $L^{2}\left(\Omega, d \mu_{\varepsilon}\right)$, then

$$
\liminf _{\varepsilon \rightarrow 0} \int_{\Omega}\left|v_{\varepsilon}\right|^{2} d \mu_{\varepsilon} \geq \int_{\Omega} v^{2} d \mu .
$$

(c) Criterium of strong convergence: $v_{\varepsilon} \rightarrow v$ if and only if $v_{\varepsilon} \rightarrow v$ in $L^{2}\left(\Omega, d \mu_{\varepsilon}\right)$ and

$$
\lim _{\varepsilon \rightarrow 0} \int_{\Omega}\left|v_{\varepsilon}\right|^{2} d \mu_{\varepsilon}=\int_{\Omega} v^{2} d \mu .
$$

Let us recall some well-known results concerning the convergence in the variable space $L^{2}\left(\Omega, d \mu_{\varepsilon}\right)$. 
Lemma 2.2. $[10,13,15]$ If $\left\{\rho^{\varepsilon}\right\}_{\varepsilon>0}$ is non-degenerate perturbation of the weight function $\rho(x) \geq 0$, then:

(A1) $\left(\left(\rho^{\varepsilon}\right)^{-1}\right) \rightarrow \rho^{-1}$ in $L^{2}\left(\Omega, \rho^{\varepsilon} d x\right)$.

(A2) $\left[v_{\varepsilon} \rightarrow v\right.$ in $\left.L^{2}\left(\Omega, \rho^{\varepsilon} d x\right)\right] \Rightarrow\left[v_{\varepsilon} \rightarrow v\right.$ in $\left.L^{1}(\Omega)\right]$.

(A3) If a sequence $\left\{v_{\varepsilon} \in L^{2}\left(\Omega, \rho^{\varepsilon} d x\right)\right\}_{\varepsilon>0}$ is bounded, then the weak convergence $v_{\varepsilon} \rightarrow v$ in $L^{2}\left(\Omega, \rho^{\varepsilon} d x\right)$ is equivalent to the weak convergence $\rho^{\varepsilon} v_{\varepsilon} \rightarrow \rho v$ in $L^{1}(\Omega)$.

(A4) If $a \in L^{\infty}$ and $v_{\varepsilon} \rightarrow v$ in $L^{2}\left(\Omega, \rho^{\varepsilon} d x\right)$, then $a v_{\varepsilon} \rightarrow a v$ in $L^{2}\left(\Omega, \rho^{\varepsilon} d x\right)$.

Variable Sobolev spaces. Let $\rho(x)$ be a degenerate weight function and let $\left\{\rho^{\varepsilon}\right\}_{\varepsilon>0}$ be a non-degenerate perturbation of the function $\rho$ in the sense of Definition 2.2. We denote by $H\left(\Omega, \rho^{\varepsilon} d x\right)$ the closure of $C_{0}^{\infty}(\Omega)$ with respect to the norm $\|\cdot\|_{\rho^{\varepsilon}}$. Since for every $\varepsilon$ the function $\rho^{\varepsilon}$ is non-degenerate, that is, $\rho^{\varepsilon}$ is bounded between two positive constants, the space $H\left(\Omega, \rho^{\varepsilon} d x\right)$ (and the spaces $L^{2}\left(\Omega, \rho^{\varepsilon} d x\right)$ and $L^{2}\left(\Omega,\left(\rho^{\varepsilon}\right)^{-1} d x\right)$ ) coincides with the classical Sobolev space $H_{0}^{1}(\Omega)$ (with $\left.L^{2}(\Omega)\right)$.

Definition 2.3. We say that a sequence $\left\{y_{\varepsilon} \in H\left(\Omega, \rho^{\varepsilon} d x\right)\right\}_{\varepsilon>0}$ converges weakly to an element $y \in W$ as $\varepsilon \rightarrow 0$, if the following hold: (i) This sequence is bounded. (ii) $y_{\varepsilon} \rightarrow y$ in $L^{2}\left(\Omega, \rho^{\varepsilon} d x\right)$. (iii) $\nabla y_{\varepsilon} \rightarrow \nabla y$ in $L^{2}\left(\Omega, \rho^{\varepsilon} d x\right)^{N}$.

Compensated Compactness Lemma in variable Lebesgue and Sobolev spaces. Let $p, q$ such that $2 \leq p<\infty, 1 / p+1 / q=1$ and let $\left\{\rho^{\varepsilon}\right\}_{\varepsilon>0}$ be a non-degenerate perturbation of a weight function $\rho$. We associate to every $\rho^{\varepsilon}$ the space

$$
X\left(\Omega, \rho^{\varepsilon} d x\right)=\left\{\vec{f} \in L^{q}\left(\Omega, \rho^{\varepsilon} d x\right)^{N} \mid \operatorname{div}\left(\rho^{\varepsilon} \vec{f}\right) \in L^{q}(\Omega)\right\} \forall \varepsilon>0
$$

with the norm

$$
\|\vec{f}\|_{X\left(\Omega, \rho^{\varepsilon} d x\right)}=\left(\|\vec{f}\|_{L^{q}\left(\Omega, \rho^{\varepsilon} d x\right)^{N}}^{q}+\left\|\operatorname{div}\left(\rho^{\varepsilon} \vec{f}\right)\right\|_{L^{q}(\Omega)}^{q}\right)^{1 / q} .
$$

We say that a sequence $\left\{\vec{f}_{\varepsilon} \in X\left(\Omega, \rho^{\varepsilon} d x\right)\right\}_{\varepsilon>0}$ is bounded if

$$
\limsup _{\varepsilon \rightarrow 0}\left\|\vec{f}_{\varepsilon}\right\|_{X\left(\Omega, \rho^{\varepsilon} d x\right)}<+\infty .
$$

In order to discuss the problem of $\mathrm{H}$-attainability we need the following result.

Lemma 2.3. [3] Let $\left\{\rho^{\varepsilon}\right\}_{\varepsilon>0}$ be a non-degenerate perturbation of a weight function $\rho(x)>0$. Let $\left\{\vec{f} \in L^{q}\left(\Omega, \rho^{\varepsilon} d x\right)^{N}\right\}_{\varepsilon>0}$ and $\left\{g_{\varepsilon \in H\left(\Omega, \rho^{\varepsilon} d x\right)}\right\}_{\varepsilon>0}$ be sequences such that $\left\{\vec{f}_{\varepsilon}\right\}_{\varepsilon>0}$ is bounded in the variable space $X\left(\Omega, \rho^{\varepsilon} d x\right), \vec{f}_{\varepsilon} \rightarrow \vec{f}$ weakly in $L^{q}\left(\Omega, \rho^{\varepsilon} d x\right)^{N},\left\{g_{\varepsilon}\right\}_{\varepsilon>0}$ is bounded in the variable space $H\left(\Omega, \rho^{\varepsilon} d x\right), g_{\varepsilon} \rightarrow g$ in $L^{p}(\Omega)$, and $\nabla g_{\varepsilon} \rightarrow \nabla g$ in $L^{p}\left(\Omega, \rho^{\varepsilon} d x\right)^{N}$. Then

$$
\lim _{\varepsilon \rightarrow 0} \int_{\Omega} \varphi\left(\vec{f}_{\varepsilon}, \nabla g_{\varepsilon}\right)_{\mathbb{R}^{N}} \rho^{\varepsilon} d x=\int_{\Omega} \varphi(\vec{f}, \nabla g)_{\mathbb{R}^{N}} \rho d x, \forall \varphi \in C_{0}^{\infty}(\Omega) .
$$


Further, we consider a special "lifting" operator

$$
T_{\varepsilon}: L^{p}(\Omega, \rho d x) \rightarrow L^{p}\left(\Omega, \rho^{\varepsilon} d x\right)
$$

defined as follows

$$
\int_{\Omega} T_{\varepsilon} y \varphi \rho^{\varepsilon} d x=\int_{\Omega} y(\varphi)_{\varepsilon} \rho d x \forall \varphi \in C_{0}^{\infty}(\Omega), \forall \varepsilon>0 .
$$

Firstly this operator was constructed in [14] for the case of an arbitrary measure. Let us consider the following well-known result.

Lemma 2.4. [10, Lemma 7.2] Let $\rho \in L_{\text {loc }}^{1}\left(\mathbb{R}^{N}\right)$ be a degenerate weight function and let $\left\{\rho^{\varepsilon}=(\rho)_{\varepsilon}\right\}_{\varepsilon>0}$ be a "direct" smoothing of $\rho$. Then for every element $y \in L^{p}(\Omega, \rho d x)$ there exists a sequence $\left\{T_{\varepsilon} y \in L^{p}\left(\Omega, \rho^{\varepsilon} d x\right)\right\}_{\varepsilon>0}$ such that $T_{\varepsilon} y \rightarrow y$ in $L^{p}\left(\Omega, \rho^{\varepsilon} d x\right)$.

Let us recall that a function $a \in L^{2}(\Omega, \rho d x)$ and a vector $b \in L^{2}(\Omega, \rho d x)^{N}$ are related by the equality

$$
\operatorname{div}(\rho b)=a \text { if } \int_{\Omega}(b, \nabla \varphi)_{\mathbb{R}^{N}} \rho d x=-\int_{\Omega} a \varphi \rho d x \forall \varphi \in C_{0}^{\infty}(\Omega) .
$$

In a similar way, for $a^{\varepsilon} \in L^{2}\left(\Omega, \rho^{\varepsilon} d x\right)$ and $b \in L^{2}\left(\Omega, \rho^{\varepsilon} d x\right)^{N}$, we have

$$
\operatorname{div}\left(\rho^{\varepsilon} b^{\varepsilon}\right)=a^{\varepsilon} \text { if } \int_{\Omega}\left(b^{\varepsilon}, \nabla \varphi\right)_{\mathbb{R}^{N}} \rho^{\varepsilon} d x=-\int_{\Omega} a^{\varepsilon} \varphi \rho^{\varepsilon} d x \forall \varphi \in C_{0}^{\infty}(\Omega) .
$$

Note that by arguments of completion, the above identities can be extended to test functions from $H$ and $H\left(\Omega, \rho^{\varepsilon} d x\right)$, respectively.

Lemma 2.5. [10, Lemma 7.3] If $a \in L^{2}(\Omega, \rho d x)$ and $b \in L^{2}(\Omega, \rho d x)^{N}$ are related by (2.16), then $a^{\varepsilon}=T_{\varepsilon} a$ and $b^{\varepsilon}=T_{\varepsilon} b$ are related by (2.17).

Following $[10,11]$ we can give a dual description of the weighted Sobolev space $H$. Let us consider two spaces: the first is $X_{\rho}^{2}$ as the closure of the set $\{(y, \nabla y), y \in$ $\left.C_{0}^{\infty}(\Omega)\right\}$ in $L^{2}(\Omega, \rho d x) \times L^{2}(\Omega, \rho d x)^{N}$, hence, the elements of this space are pairs $(y, v)$, where $y$ is a function in $H$ and $v=\nabla y$ is its gradient. The second space $\tilde{X}_{\rho}^{2}$ consists of pairs $(y, v)$, where $y \in L^{2}(\Omega, \rho d x)$ abd $v \in L^{2}(\Omega, \rho d x)^{N}$ are such that

$$
\int_{\Omega} y a \rho d x=-\int_{\Omega}(v, b)_{\mathbb{R}^{N}} \rho d x
$$

for any $(a, b)$ satisfying the conditions

$$
a \in L^{2}(\Omega, \rho d x), b \in L^{2}(\Omega, \rho d x)^{N}, a=\operatorname{div}(\rho b)
$$

It is easy to see that $X_{\rho}^{2}$ and $\tilde{X}_{\rho}^{2}$ are closed in $L^{2}(\Omega, \rho d x)^{N+1}$ and $X_{\rho}^{2} \subseteq \tilde{X}_{\rho}^{2}$. Moreover, from [10, Lemma 7.4] (or [11, Theorem 1]) we have that $X_{\rho}^{2}=\tilde{X}_{\rho}^{2}$.

The next Theorem establishes the possibility of passing to the limit as $\varepsilon \rightarrow 0$ in variable space $H\left(\Omega, \rho^{\varepsilon} d x\right)$. 
Theorem 2.3. [10, Theorem 7.1] Let $\rho^{\varepsilon}=(\rho)_{\varepsilon}$ be a direct smoothing of a degenerate weight $\rho \in L_{l o c}^{1}\left(\mathbb{R}^{N}\right)$ and let $y^{\varepsilon} \in H\left(\Omega, \rho^{\varepsilon} d x\right), y^{\varepsilon} \rightarrow y$ in $L^{2}\left(\Omega, \rho^{\varepsilon} d x\right)$, $\nabla y^{\varepsilon} \rightarrow v$ in $L^{2}\left(\Omega, \rho^{\varepsilon} d x\right)^{N}$. Then $y \in H$ and $v=\nabla y$.

\section{Setting of the Optimal Control Problem}

Let $K$ be a non-empty convex closed subset of the space $W$, and let $K$ be sequentialy closed with respect to the norm

$$
\|y\|^{2}:=\int_{\Omega} y^{2} \rho d x+\int_{\Omega}\left|\nabla y+\frac{y}{2} \nabla \ln \rho\right|_{\mathbb{R}^{N}}^{2} \rho d x .
$$

Let $y_{a d} \in L^{2}(\Omega), f \in L^{2}\left(\Omega, \rho^{-1} d x\right)$ and $u_{0} \in L^{2}\left(\Omega, \rho^{-1} d x\right)$ be given distribution, and $U_{\partial}$ be a non-empty convex closed subset in $L^{2}\left(\Omega, \rho^{-1} d x\right)$ such that

$$
U_{\partial}=\left\{u \in L^{2}\left(\Omega, \rho^{-1} d x\right):\left\|u-u_{0}\right\|_{L^{2}\left(\Omega, \rho^{-1} d x\right)} \leq R\right\} .
$$

Hereinafter functions $u \in U_{\partial}$ are considered to be admissible controls.

The main object we deal with in the paper is the following optimal control problem for the variational inequality with control in the right hand side:

$$
\begin{gathered}
I(u, y)=\frac{1}{2}\left\|y-y_{a d}\right\|_{L^{2}(\Omega, \rho d x)}^{2} \rightarrow \text { inf }, \\
u \in U_{\partial}, y \in K, \\
\int_{\Omega}(\nabla y, \nabla v-\nabla y)_{\mathbb{R}^{N}} \rho d x \geq \int_{\Omega}(f+u)(v-y) d x, \quad \forall v \in K .
\end{gathered}
$$

Let us consider the following linear operator related to the variational inequality (3.5):

$$
A: W_{0}^{1,2}(\Omega ; \rho d x) \rightarrow\left(W_{0}^{1,2}(\Omega ; \rho d x)\right)^{*},
$$

that is defined by the rule:

$$
\langle A y, v-y\rangle_{H(\Omega ; \rho d x)}=\int_{\Omega}(\nabla y, \nabla v-\nabla y)_{\mathbb{R}^{N}} \rho d x \quad \forall v \in K .
$$

Here

$$
\langle\cdot, \cdot\rangle_{H(\Omega ; \rho d x)}:(H(\Omega ; \rho d x))^{*} \times H(\Omega ; \rho d x) \rightarrow \mathbb{R}
$$

is the duality pairing. It is clear that

$$
A y=-\operatorname{div}(\rho(x) \nabla y) .
$$

Similarly to [4] let us consider the next definitions.

Definition 3.1. We say that a function $y=y(u, f) \in K$ is a $W$-solution to degenerate variational inequality (3.4)-(3.5) if

$$
\langle-\operatorname{div}(\rho(x) \nabla y), v-y\rangle_{W} \geq\langle f+u, v-y\rangle_{W}
$$

holds for any $v \in K$. 
Definition 3.2. Let $\tilde{K}$ be a closure in the space $C_{0}^{\infty}(\Omega)$ of the set $K \cap C_{0}^{\infty}(\Omega)$. We say that a function $y=y(u, f) \in \tilde{K}$ is an H-solution to variational inequality (3.4)-(3.5) if

$$
\langle-\operatorname{div}(\rho(x) \nabla y), v-y\rangle_{H(\Omega ; \rho d x)} \geq\langle f+u, v-y\rangle_{H(\Omega ; \rho d x)}
$$

holds for any $v \in \tilde{K}$.

Remark 3.1. It is easy to say that the set $\tilde{K} \subset H$ is closed and convex.

Let us remark that in the case when the function $\rho$ is a weight function of potential type in the sense of Definition 2.1 we can prove the existence and uniqueness of $W$-solution for the inequality (3.4)-(3.5), namely the following result takes place:

Theorem 3.1. [17, Tеорема 2] Let $\rho: \Omega \rightarrow \mathbb{R}_{+}$be a weight function of potential type. Then for given $f \in L^{2}\left(\Omega, \rho^{-1} d x\right)$ and $u \in U_{\partial}$ the variational inequality (3.4)(3.5) has unique solution $y=y(u, f) \in K$ such that $y=z / \sqrt{\rho}$ and $z \in H_{0}^{1}(\Omega)$.

Remark 3.2. Similar result with Theorem 3.1 concerning existence and uniqueness of $H$-solution to problem (3.4)-(3.5) can be easily obtained using similar argumentation.

Taking this fact into account we can introduce two sets of admissible pairs to the optimal control problem (3.3)-(3.5):

$$
\begin{aligned}
& \Xi_{W}=\left\{(u, y) \in U_{\partial} \times W \mid y \in K,(u, y) \text { are related by }(3.6)\right\}, \\
& \Xi_{H}=\left\{(u, y) \in U_{\partial} \times H \mid y \in \tilde{K},(u, y) \text { are related by (3.7) }\right\} .
\end{aligned}
$$

Hence for the given control object described by relations (3.4)-(3.5) with both fixed control constrains $\left(u \in U_{\partial}\right)$ and fixed cost functional (3.3), we have two different statement of the original optimal control problem, namely

$$
\left\langle\inf _{(u, y) \in \Xi_{W}} I(u, y)\right\rangle \text { and }\left\langle\inf _{(u, y) \in \Xi_{H}} I(u, y)\right\rangle \text {. }
$$

Having assumed that $W \neq H$ for a given degenerate weight function $\rho \geq 0$, we can come to the effect which is usually called the Lavrentieff phenomenon. It means that for some $u \in U_{\partial}$ and $f \in L^{2}\left(\Omega, \rho^{-1} d x\right)$ an $H$-solution to problem (3.4)-(3.5) does not coincide with its $W$-solution [13].

Remark 3.3. In view of Theorem 3.1 and Remark 3.2, the set $\Xi_{H}$ is always nonempty.

Let us consider the following concept.

Definition 3.3. We say that a pair $\left(u^{0}, y^{0}\right) \in L^{2}\left(\Omega, \rho^{-1} d x\right) \times H$ is an $H$-optimal solution to problem (3.3)-(3.5) if $\left(u^{0}, y^{0}\right) \in \Xi_{H}$ and

$$
I\left(u^{0}, y^{0}\right)=\inf _{(u, y) \in \Xi_{H}} I(u, y)
$$


Note that optimal control problem (3.3)-(3.5) is solvable, namely the following result takes place.

Theorem 3.2. Let $\rho(x)>0$ be a degenerate weight function of potential type. Then the set of H-optimal solutions to problem (3.3)-(3.5) is non-empty $\forall f \in$ $L^{2}\left(\Omega, \rho^{-1} d x\right)$.

\section{Attainability of $H$-optimal Solutions}

In this section we propose a regular algorithm of approximation (perturbation) for the original degenerate optimal control problem (3.3)-(3.5) and it will be shown that $H$-optimal solutions of mentioned problem can be attained by optimal solutions of perturbed problems. Note that in view of Theorem 3.2 that the set of $H$-optimal solutions to the problem (3.3)-(3.5) is non-empty.

Let $\rho$ be a degenerate weight function with properties (2.2)-(2.1), and let $\{\rho \varepsilon\}_{\varepsilon>0}$ be a non-degenerate perturbation of $\rho$ in the sense of Definition 2.2

Definition 4.1. We say that a bounded sequence

$$
\left\{\left(u_{\varepsilon}, y_{\varepsilon}\right) \in \mathbb{Y}\left(\Omega, \rho^{\varepsilon} d x\right)=L^{2}\left(\Omega,\left(\rho^{\varepsilon}\right)^{-1} d x\right) \times H\left(\Omega, \rho^{\varepsilon} d x\right)\right\}_{\varepsilon>0}
$$

$w$-converges to $(u, y) \in L^{2}\left(\Omega, \rho^{-1} d x\right) \times W$ in the variable space $\mathbb{Y}\left(\Omega, \rho^{\varepsilon} d x\right)$ as $\varepsilon \rightarrow 0$, if $u_{\varepsilon} \rightarrow u$ in $L^{2}\left(\Omega,\left(\rho^{\varepsilon}\right)^{-1} d x\right), y_{\varepsilon} \rightarrow y$ in $L^{2}\left(\Omega, \rho^{\varepsilon} d x\right), \nabla y_{\varepsilon} \rightarrow \nabla y$ in $L^{2}\left(\Omega, \rho^{\varepsilon} d x\right)^{N}$.

Definition 4.2. We say that a minimization problem

$$
\left\langle\inf _{(u, y) \in \Xi_{H}} I(u, y)\right\rangle
$$

is a weak variational limit (or variational $w$-limit) of the sequence

$$
\left\{\left\langle\inf _{\left(u_{\varepsilon}, y_{\varepsilon}\right) \in \Xi_{\varepsilon}} I_{\varepsilon}\left(u_{\varepsilon}, y_{\varepsilon}\right)\right\rangle ; \Xi_{\varepsilon} \subset \mathbb{Y}\left(\Omega, \rho^{\varepsilon} d x\right), \varepsilon>0\right\},
$$

with respect to $w$-convergence in variable space $\mathbb{Y}\left(\Omega, \rho^{\varepsilon} d x\right)$, if the following conditions are satisfied:

(1) if $\left\{\varepsilon_{k}\right\}$ is a subsequence of $\{\varepsilon\}$ such that $\varepsilon_{k} \rightarrow 0$ as $k \rightarrow \infty$, and a sequence $\left\{\left(u_{k}, y_{k}\right) \in \Xi_{\varepsilon_{k}}\right\}_{\varepsilon>0} w$-converges to a pair $(u, y)$, then

$$
(u, y) \in \Xi_{H} ; I(u, y) \leq \liminf _{k \rightarrow \infty} I_{\varepsilon_{k}}\left(u_{k}, y_{k}\right) ;
$$

(2) for every pair $(u, y) \in \Xi_{H}$ and any value $\delta>0$ there exists a realizing sequence $\left\{\left(\hat{u}_{\varepsilon}, \hat{y}_{\varepsilon}\right) \in \mathbb{Y}\left(\Omega, \rho^{\varepsilon} d x\right)\right\}_{\varepsilon>0}$ such that

$$
\begin{gathered}
\left(\hat{u}_{\varepsilon}, \hat{y}_{\varepsilon}\right) \in \Xi_{\varepsilon} \forall \varepsilon>0,\left(\hat{u}_{\varepsilon}, \hat{y}_{\varepsilon}\right) w-\text { converges to }(\hat{u}, \hat{y}), \\
\|u-\hat{u}\|_{L^{2}\left(\Omega, \rho^{-1} d x\right)}+\|y-\hat{y}\|_{\rho} \leq \delta, I(u, y) \geq \underset{\varepsilon \rightarrow 0}{\limsup I_{\varepsilon}\left(\hat{u}_{\varepsilon}, \hat{y}_{\varepsilon}\right)-\delta .}
\end{gathered}
$$


The last definition is motivated by the following property of variational $w$ limits (for the details we refer to [2]).

Theorem 4.1. Assume that (4.1) is a weak variational limit of the sequence (4.2), and the constrained minimization problem (4.1) has a solution. Suppose $\left\{\left(u_{\varepsilon}^{0}, y_{\varepsilon}^{0}\right) \in \Xi_{\varepsilon}\right\}$ is a sequence of optimal pairs to (4.2). Then there exists a pair $\left(u^{0}, y^{0}\right) \in \Xi_{H}$ such that $\left(u_{\varepsilon}^{0}, y_{\varepsilon}^{0}\right) w$-converges to $\left(u^{0}, y^{0}\right)$, and

$$
\inf _{(u, y) \in \Xi_{H}} I(u, y)=I\left(u^{0}, y^{0}\right)=\lim _{\varepsilon \rightarrow 0} \inf _{\left(u_{\varepsilon}, y_{\varepsilon}\right) \in \Xi_{\varepsilon}} I_{\varepsilon}\left(u_{\varepsilon}, y_{\varepsilon}\right) .
$$

Let us consider the sequences $\left\{K_{\varepsilon}\right\}_{\varepsilon>0}$ and $\left\{U_{\partial}^{\varepsilon}\right\}_{\varepsilon>0}$ of non-empty convex closed subsets, which sequentially converges to sets $\tilde{K}$ and $U_{\partial}$, respectively, in the sense of Kuratovski as $\varepsilon \rightarrow 0$ with respect to weak topology of spaces $H\left(\Omega, \rho^{\varepsilon} d x\right)$ and $L^{2}\left(\Omega,\left(\rho^{\varepsilon}\right)^{-1} d x\right)$, respectively, and let Hypothesis 2 hold true for $X=L^{2}\left(\Omega,\left(\rho^{\varepsilon}\right)^{-1} d x\right)$ and $V=H\left(\Omega, \rho^{\varepsilon} d x\right) \forall \varepsilon>0$. Taking into account Theorem 4.1 , we consider the following collection of perturbed optimal control problems for non-degenerate elliptic variational inequalities:

$$
\begin{gathered}
\text { Minimize }\left\{I_{\varepsilon}(u, y)=\frac{1}{2} \int_{\Omega}\left|y(x)-y_{a d}\right|^{2} d x\right\}, \\
u \in U_{\partial}^{\varepsilon}, y \in K_{\varepsilon}, \\
\left\langle-\operatorname{div}\left(\rho^{\varepsilon}(x) \nabla y\right), v-y\right\rangle_{H\left(\Omega ; \rho^{\varepsilon} d x\right)} \geq\langle f+u, v-y\rangle_{H\left(\Omega ; \rho^{\varepsilon} d x\right)} \forall v \in K_{\varepsilon},
\end{gathered}
$$

where the elements $y_{a d} \in L^{2}(\Omega), f \in L^{2}\left(\Omega, \rho^{-1} d x\right) \subset L^{2}\left(\Omega,\left(\rho^{\varepsilon}\right)^{-1} d x\right)$ are the same as for original problem (3.3)-(3.5). For every $\varepsilon>0$ we define $\Xi_{\varepsilon}$ as a set of all admissible pairs to the problem (4.6)-(4.8), namely $(u, y) \in \Xi_{H}$ if and only if the pair $(u, y)$ satisfies (4.7)-(4.8).

Let us discuss the optimality conditions for problem (4.6)-(4.8). Let $V=$ $H\left(\Omega, \rho^{\varepsilon} d x\right), H=L^{2}(\Omega)$. Taking into account suggestions of the section 2 , we have that $V$ and $H$ are Hilbert spaces, and $V \hookrightarrow H$ continuously and $V$ is dense in $H$. Let us denote by $(\cdot, \cdot)$ the scalar product in $H$. Let us identify $H$ with its conjugated $H^{*}$, and let $V^{*}$ be the space conjugated to $V$. Then $V \subset H \subset V^{*}$ and every space is dense in the next one and corresponding embeddings are continuous. Let $U=L^{2}\left(\Omega,\left(\rho^{\varepsilon}\right)^{-1} d x\right)$ be the control space (which coincides with $L^{2}(\Omega)$ ), $U_{\partial}^{\varepsilon}$ is convex and closed in $U$ by the construction. Let us consider an operator $A: V \rightarrow V^{*}, A y=-\operatorname{div}\left(\rho^{\varepsilon}(x) \nabla y\right)$, and functions $f$ and $y_{a d}$ as in previous suggestions. For every control $u \in U$ the state $y(u)$ is defined as the solution to the following problem

$$
A y=f+u, y \in H\left(\Omega, \rho^{\varepsilon} d x\right) .
$$

Let us consider for every $u \in U$ the cost functional

$$
J(u, y)=\frac{1}{2}\left\|y(u)-y_{a d}\right\|_{H}^{2} .
$$


The optimal control problem is to find such pair $(u, y) \in U_{\partial}^{\varepsilon} \times H\left(\Omega, \rho^{\varepsilon} d x\right)$ that

$$
J(u, y)=\inf _{(v, y(v)) \in U_{\partial}^{\varepsilon} \times H\left(\Omega, \rho^{\varepsilon} d x\right)} J(v, y(v)) \text { with conditions (4.9). }
$$

It is known that the solution of the optimal control problem is characterized by the inequality

$$
J_{u}^{\prime}(u, y(u))(v-u) \geq 0, \forall v \in U_{\partial}^{\varepsilon} .
$$

Since, $A$ is an isomorphism of the space $V$ to $V^{*}$ (see for details [8]), then $y(u)=$ $A^{-1}(f+u)$, and then

$$
y^{\prime}(u)(v-u)=A^{-1}(v-u)=y(v)-y(u) .
$$

Hence, (4.12) is equivalent to the following inequality:

$$
\left(y(u)-y_{a d}, y(v)-y(u)\right) \geq 0, \forall v \in U_{\partial}^{\varepsilon} .
$$

Let $A^{*} \in \mathcal{L}\left(V, V^{*}\right)$ be the conjugate operator to $A$ and it is an isomorphism of $V$ on $V^{*}$ as well as $A$. For the control $v \in U_{\partial}^{\varepsilon}$ let us define the conjugate state $p(v) \in V$ by the next relation:

$$
A^{*} p(v)=y(v)-y_{a d} .
$$

Then

$$
\begin{aligned}
\left(A^{*} p(u), y(v)-y(u)\right) & =\left(y(u)-y_{a d}, y(v)-y(u)\right)=(p(u), A y(v)-A y(u)) \\
=(p(u), v-u) & =(p(u), v-u)_{U}=\int_{\Omega} p(u)(v-u) d x \geq 0,
\end{aligned}
$$

since $p(u) \in V \subset L^{2}\left(\Omega, \rho^{\varepsilon} d x\right), v-u \in L^{2}\left(\Omega,\left(\rho^{\varepsilon}\right)^{-1} d x\right)$. Similarly to [1, Theorem 1.4], obtained results can be formulated as the following theorem.

Theorem 4.2. Let $a(u, v)=(A u, v)$ be a bilinear continuous and coercive form on $V$, and cost functional be as in (4.10). The element $u \in U_{\partial}^{\varepsilon}$ is the optimal control if and only if the following relations are fulfilled:

$$
\begin{gathered}
-\operatorname{div}\left(\rho^{\varepsilon}(x) y\right)=f+u \quad \text { in } \Omega, y \in V, \\
-\operatorname{div}\left(\rho^{\varepsilon}(x) p\right)=y-y_{a d} \quad \text { in } \Omega, p \in V, \\
\int_{\Omega} p(u)(v-u) d x \geq 0, \forall v \in U_{\partial}^{\varepsilon} .
\end{gathered}
$$

Remark 4.1. Let us recall that sequential $K$-upper and $K$-lower limits of a sequence of sets $\left\{E_{k}\right\}_{k \in \mathbb{N}}$ are defined as follows, respectively:

$$
\begin{gathered}
K_{s}-\overline{\lim } E_{k}=\left\{y \in X: \exists \sigma(k) \rightarrow \infty, \exists y_{k} \rightarrow y, \forall k \in \mathbb{N}: y_{k} \in E_{\sigma(k)}\right\}, \\
K_{s}-\underline{\lim } E_{k}=\left\{y \in X: \exists y_{k} \rightarrow y \exists k \geq k_{0} \in \mathbb{N}: y_{k} \in E_{k}\right\} .
\end{gathered}
$$

The sequence $\left\{E_{k}\right\}_{k \in \mathbb{N}}$ sequantially converges in the sense of Kuratovski to the set $E$ (shortly, $K_{s}$-converges), if $E=K_{s}-\underline{\lim } E_{k}=K_{s}-\overline{\lim } E_{k}$. 
Lemma 4.1. Let $\left\{\rho^{\varepsilon}=(\rho)_{\varepsilon}\right\}_{\varepsilon>0}$ be a "direct" smoothing of a degenerate weight function $\rho \geq 0$. Let $\left\{\left(u_{\varepsilon}, y_{\varepsilon}\right) \in \Xi_{\varepsilon}\right\}_{\varepsilon>0}$ be a sequence of admissible pairs to the problem (4.6)-(4.8). Then there exists a pair $\left\{\left(u^{*}, y^{*}\right)\right\}$ and a subsequence $\left\{\left(u_{\varepsilon_{k}}, y_{\varepsilon_{k}}\right)\right\}_{k \in \mathbb{N}}$ of $\left\{\left(u_{\varepsilon}, y_{\varepsilon}\right) \in \Xi_{\varepsilon}\right\}_{\varepsilon>0}$ such that $\left(u_{\varepsilon_{k}}, y_{\varepsilon_{k}}\right)$ w-converges to $\left\{\left(u^{*}, y^{*}\right)\right\}$ as $k \rightarrow \infty$ and $\left(u^{*}, y^{*}\right) \in \Xi_{H}$.

Proof. Let us consider the following variational inequality:

$$
\left\langle-\operatorname{div}\left(\rho^{\varepsilon} \nabla y_{\varepsilon}\right), v_{\varepsilon}-y_{\varepsilon}\right\rangle_{H\left(\Omega, \rho^{\varepsilon} d x\right)} \geq\left\langle f+u_{\varepsilon}, v_{\varepsilon}-y_{\varepsilon}\right\rangle_{H\left(\Omega, \rho^{\varepsilon} d x\right)}, \forall v_{\varepsilon} \in K_{\varepsilon} .
$$

Let us show the bondedness of the sequence $\left\{y_{\varepsilon}\right\}_{\varepsilon>0}$ in the space $H\left(\Omega, \rho^{\varepsilon} d x\right)$. Let us suppose that $\left\|y_{\varepsilon}\right\|_{H\left(\Omega, \rho^{\varepsilon} d x\right)} \rightarrow \infty$ as $\varepsilon \rightarrow 0$. Then on the one hand

$$
\begin{gathered}
\left\langle-\operatorname{div}\left(\rho^{\varepsilon} \nabla y_{\varepsilon}\right), y_{\varepsilon}-v_{\varepsilon}\right\rangle_{H\left(\Omega, \rho^{\varepsilon} d x\right)} \\
\left\|f+u_{\varepsilon}\right\|_{L^{2}\left(\Omega,\left(\rho^{\varepsilon}\right)^{-1} d x\right)}\left\|y_{\varepsilon}-v_{\varepsilon}\right\|_{L^{2}\left(\Omega, \rho^{\varepsilon} d x\right)} \\
\leq\left\|f+u_{\varepsilon}\right\|_{L^{2}\left(\Omega,\left(\rho^{\varepsilon}\right)^{-1} d x\right)}\left\|y_{\varepsilon}-v_{\varepsilon}\right\|_{H\left(\Omega, \rho^{\varepsilon} d x\right)}, \forall v_{\varepsilon} \in K_{\varepsilon}, \forall \varepsilon>0 .
\end{gathered}
$$

On the other hand, for arbitrary fixed element $v \in \tilde{K}$ let us consider the sequence $\left\{v_{\varepsilon} \in K_{\varepsilon}\right\}_{\varepsilon>0}$ such that $v_{\varepsilon} \rightarrow v$ in $H\left(\Omega, \rho^{\varepsilon} d x\right)$ (note, that such sequence always exists provided $\left.\tilde{K}=K_{s}-\lim K_{\varepsilon}\right)$, and taking into account the definition and properties of the space $H\left(\Omega, \rho^{\varepsilon} d x\right)$ and operator $A: H\left(\Omega, \rho^{\varepsilon} d x\right) \rightarrow\left(H\left(\Omega, \rho^{\varepsilon} d x\right)\right)^{*}$, $A y_{\varepsilon}=-\operatorname{div}\left(\rho^{\varepsilon} \nabla y_{\varepsilon}\right)$, we obtain such estimations:

$$
\begin{gathered}
\left\langle A y_{\varepsilon}, y_{\varepsilon}\right\rangle_{H\left(\Omega, \rho^{\varepsilon} d x\right)}=\int_{\Omega}\left(\nabla y_{\varepsilon}, \nabla y_{\varepsilon}\right)_{\mathbb{R}^{N}} \rho^{\varepsilon} d x \geq C_{1}\left\|y_{\varepsilon}\right\|_{H\left(\Omega, \rho^{\varepsilon} d x\right)}^{2}, C_{1}>0, \\
\left\langle A y_{\varepsilon}, y_{\varepsilon}-v_{\varepsilon}\right\rangle_{H\left(\Omega, \rho^{\varepsilon} d x\right)} \geq C_{1}\left\|y_{\varepsilon}\right\|_{H\left(\Omega, \rho^{\varepsilon} d x\right)}^{2}-\left\|\nabla y_{\varepsilon}\right\|_{L^{2}\left(\Omega, \rho^{\varepsilon} d x\right)^{N}}\left\|\nabla v_{\varepsilon}\right\|_{L^{2}\left(\Omega, \rho^{\varepsilon} d x\right)^{N} .}
\end{gathered}
$$

Hence, we have the following relations

$$
\begin{gathered}
\frac{\left\langle-\operatorname{div}\left(\rho^{\varepsilon \nabla y_{\varepsilon}}\right), y_{\varepsilon}-v_{\varepsilon}\right\rangle_{H\left(\Omega, \rho^{\varepsilon} d x\right)}}{\left\|y_{\varepsilon}-v_{\varepsilon}\right\|_{H\left(\Omega, \rho^{\varepsilon} d x\right)}} \\
\geq \frac{C_{1}\left\|y_{\varepsilon}\right\|_{H\left(\Omega, \rho^{\varepsilon} d x\right)}^{2}-\left\|\nabla y_{\varepsilon}\right\|_{L^{2}\left(\Omega, \rho^{\varepsilon} d x\right)^{N}}\left\|\nabla v_{\varepsilon}\right\|_{L^{2}\left(\Omega, \rho^{\varepsilon} d x\right)^{N}}}{\left\|y_{\varepsilon}\right\|_{H\left(\Omega, \rho^{\varepsilon} d x\right)}+\left\|v_{\varepsilon}\right\|_{H\left(\Omega, \rho^{\varepsilon} d x\right)}} \\
\geq \frac{C_{1}\left\|y_{\varepsilon}\right\|_{H\left(\Omega, \rho^{\varepsilon} d x\right)}^{2}-C_{2}\left\|y_{\varepsilon}\right\|_{H\left(\Omega, \rho^{\varepsilon} d x\right)}\left\|v_{\varepsilon}\right\|_{H\left(\Omega, \rho^{\varepsilon} d x\right)}}{\left\|y_{\varepsilon}\right\|_{H\left(\Omega, \rho^{\varepsilon} d x\right)}+\left\|v_{\varepsilon}\right\|_{H\left(\Omega, \rho^{\varepsilon} d x\right)}} \\
\geq\left\|y_{\varepsilon}\right\|_{H\left(\Omega, \rho^{\varepsilon} d x\right)}\left(\frac{C_{1}\left\|y_{\varepsilon}\right\|_{H\left(\Omega, \rho^{\varepsilon} d x\right)}-C_{2}\left\|v_{\varepsilon}\right\|_{H\left(\Omega, \rho^{\varepsilon} d x\right)}}{\left\|y_{\varepsilon}\right\|_{H\left(\Omega, \rho^{\varepsilon} d x\right)}+\left\|v_{\varepsilon}\right\|_{H\left(\Omega, \rho^{\varepsilon} d x\right)}}\right) \\
=\left\|y_{\varepsilon}\right\|_{H\left(\Omega, \rho^{\varepsilon} d x\right)}\left(\frac{C_{1}-C_{2} \frac{\left\|v_{\varepsilon}\right\|_{H\left(\Omega, \rho^{\varepsilon} d x\right)}}{\left\|y_{\varepsilon}\right\|_{H\left(\Omega, \rho^{\varepsilon} d x\right)}}}{1+\frac{\left\|v_{\varepsilon}\right\|_{H\left(\Omega, \rho^{\varepsilon} d x\right)}}{\left\|y_{\varepsilon}\right\|_{H\left(\Omega, \rho^{\varepsilon} d x\right)}}}\right) \rightarrow \infty, \varepsilon \rightarrow 0, C_{2}>0
\end{gathered}
$$


since the sequence $\left\{v_{\varepsilon}\right\}_{\varepsilon>0}$ is bounded in $H\left(\Omega, \rho^{\varepsilon} d x\right)$. The obtained contradiction with (4.16) implies that $\left\{y_{\varepsilon}\right\}_{\varepsilon>0}$ is bounded in $H\left(\Omega, \rho^{\varepsilon} d x\right)$. Note that from definition of sets $U_{\partial}^{\varepsilon}$ we have that the sequence $\left\{u_{\varepsilon} \in U_{\partial}^{\varepsilon}\right\}_{\varepsilon>0}$ is bounded in the space $L^{2}\left(\Omega,\left(\rho^{\varepsilon}\right)^{-1} d x\right)$.

Hence, there exists a subsequence $\left\{\varepsilon_{k}\right\}$ of the sequence $\{\varepsilon\}$, converging to 0 and elements $u^{*} \in L^{2}\left(\Omega, \rho^{-1} d x\right), y^{*} \in L^{2}(\Omega, \rho d x), \vec{v} \in L^{2}(\Omega, \rho d x)^{N}$ such that $u_{\varepsilon_{k}} \rightarrow$ $u^{*}$ in $L^{2}\left(\Omega,\left(\rho^{\varepsilon}\right)^{-1} d x\right), y_{\varepsilon_{k}} \rightarrow y^{*}$ in $L^{2}\left(\Omega, \rho^{\varepsilon} d x\right), \nabla y_{\varepsilon_{k}} \rightarrow \vec{v}$ in $L^{2}\left(\Omega,\left(\rho^{\varepsilon}\right)^{-1} d x\right)^{N}$. By Theorem 2.3, we have that $y^{*} \in H$ and $v=\nabla y^{*}$ and, moreover, we have $y^{*} \in \tilde{K}$ and $u^{*} \in U_{\partial}$.

In order to prove the lemma, it is left to pass to the limit in the inequality (4.15) as $\varepsilon \rightarrow 0$. Let us take in Hypothesis $1 V=H\left(\Omega, \rho^{\varepsilon_{k}} d x\right), X=L^{2}(\Omega)$. In this case it is easy to see that the imbedding $X \hookrightarrow V^{*}$ is dense and continuous, and the imbedding $H\left(\Omega, \rho^{\varepsilon_{k}} d x\right) \hookrightarrow L^{2}(\Omega)$ is compact and dense (for details we refer to [7]). Since $f \in L^{2}\left(\Omega, \rho^{-1} d x\right) \subset L^{2}\left(\Omega,\left(\rho^{\varepsilon_{k}}\right)^{-1} d x\right) \subset L^{2}(\Omega)$, then in view of Theorem 2.1 we have $\operatorname{div}\left(\rho^{\varepsilon_{k}} \nabla y_{\varepsilon_{k}}\right) \in L^{2}(\Omega) \forall k \in \mathbb{N}$. Let us consider the next relation

$$
\begin{gathered}
\int_{\Omega} \operatorname{div}\left(\rho^{\varepsilon_{k}} \nabla y_{\varepsilon_{k}}\right) \varphi d x=-\int_{\Omega}\left(\nabla y_{\varepsilon_{k}}, \nabla \varphi\right)_{\mathbb{R}^{N}} \rho^{\varepsilon_{k}} d x \\
\rightarrow-\int_{\Omega}\left(\nabla y^{*}, \nabla \varphi\right)_{\mathbb{R}^{N}} \rho d x=\int_{\Omega} \operatorname{div}(\rho \nabla y) \varphi d x, \forall \varphi \in C_{0}^{\infty}(\Omega), \text { as } k \rightarrow \infty .
\end{gathered}
$$

Hence, $\operatorname{div}\left(\rho^{\varepsilon_{k}} \nabla y_{\varepsilon_{k}}\right) \rightarrow \operatorname{div}(\rho \nabla y)$ in $L^{2}(\Omega)$ so the sequence $\left\{\operatorname{div}\left(\rho^{\varepsilon_{k}} \nabla y_{\varepsilon_{k}}\right)\right\}_{k \in \mathbb{N}}$ is bounded in $L^{2}(\Omega)$.

Let us consider the sequence $g_{\varepsilon_{k}}:=v_{\varepsilon_{k}}-y_{\varepsilon_{k}}$. We know that the sequence $\left\{g_{\varepsilon_{k}}\right\}_{k \in \mathbb{N}}$ is bounded in $H\left(\Omega, \rho^{\varepsilon_{k}} d x\right)$ and $g_{\varepsilon_{k}} \rightarrow g:=v-y^{*}$ in $H\left(\Omega, \rho^{\varepsilon_{k}} d x\right)$ as $k \rightarrow \infty$, where $\left\{v_{\varepsilon_{k}} \in K_{\varepsilon_{k}}\right\}_{k \in \mathbb{N}}$ weakly converges to $v \in \tilde{K}$ in $H\left(\Omega, \rho^{\varepsilon_{k}} d x\right)$. In view of properties of spaces $L^{2}\left(\Omega, \rho^{\varepsilon_{k}} d x\right)$ we have that the sequence $\left\{g_{\varepsilon_{k}}\right\}_{k \in \mathbb{N}}$ is bounded in $L^{2}(\Omega)$ and $g_{\varepsilon_{k}} \rightarrow g:=v-y^{*}$ in $L^{2}(\Omega)$. Taking into account Lemma 2.3 we obtain

$$
\begin{aligned}
& \left\langle-\operatorname{div}\left(\rho^{\varepsilon_{k}}(x) \nabla y_{\varepsilon_{k}}\right), v_{\varepsilon_{k}}-y_{\varepsilon_{k}}\right\rangle_{H\left(\Omega, \rho^{\varepsilon} k d x\right)} \\
& \rightarrow\left\langle-\operatorname{div}(\rho(x) \nabla y), v-y^{*}\right\rangle_{H(\Omega, \rho d x)}, \text { as } \quad k \rightarrow \infty .
\end{aligned}
$$

Let us consider the right hand side of the inequality (4.15).

$$
\int_{\Omega}\left(f+u_{\varepsilon_{k}}\right)\left(v_{\varepsilon_{k}}-y_{\varepsilon_{k}}\right) d x=\int_{\Omega} f v_{\varepsilon_{k}} d x-\int_{\Omega} f y_{\varepsilon_{k}} d x+\int_{\Omega} u_{\varepsilon_{k}} v_{\varepsilon_{k}} d x-\int_{\Omega} u_{\varepsilon_{k}} y_{\varepsilon_{k}} d x .
$$

Let us represent the last term by the following way:

$$
-\int_{\Omega} u_{\varepsilon_{k}} y_{\varepsilon_{k}} d x \pm \int_{\Omega} u_{\varepsilon_{k}} y^{*} d x=-\int_{\Omega} u_{\varepsilon_{k}}\left(y_{\varepsilon_{k}}-y^{*}\right) d x-\int_{\Omega} u_{\varepsilon_{k}} y^{*} d x .
$$


Since $y_{\varepsilon_{k}} \rightarrow y^{*}$ in $L^{2}\left(\Omega, \rho^{\varepsilon_{k}} d x\right), \nabla y_{\varepsilon_{k}} \rightarrow \nabla y^{*}$ in $L^{2}\left(\Omega, \rho^{\varepsilon_{k}} d x\right)^{N}$, then

$$
\begin{gathered}
\int_{\Omega}\left|y_{\varepsilon_{k}}\right| d x \leq\left(\int_{\Omega}\left|y_{\varepsilon_{k}}\right|^{2} \rho^{\varepsilon_{k}} d x\right)^{1 / 2}\left(\int_{\Omega}\left(\rho^{\varepsilon_{k}}\right)^{-1} d x\right)^{1 / 2} \leq \tilde{C}(|\Omega|)^{1 / 2}, \\
\int_{\Omega}\left|\nabla y_{\varepsilon_{k}}\right|_{2} d x \leq\left(\int_{\Omega}\left|\nabla y_{\varepsilon_{k}}\right|^{2} \rho^{\varepsilon_{k}} d x\right)^{1 / 2}\left(\int_{\Omega}\left(\rho^{\varepsilon_{k}}\right)^{-1} d x\right)^{1 / 2} \leq \hat{C}(|\Omega|)^{1 / 2} .
\end{gathered}
$$

Therefore the sequence $\left\{y_{\varepsilon_{k}}\right\}_{k \in \mathbb{N}}$ is equi-integrable on $\Omega$ and bounded in $W_{0}^{1,1}(\Omega)$. In view of compact embedding $W_{0}^{1,1}(\Omega) \hookrightarrow L^{1}(\Omega)$, there exists an element $\tilde{y}$ such that $y_{\varepsilon_{k}} \rightarrow \tilde{y}$ strongly in $L^{1}(\Omega)$. However, it is easy to see that $y_{\varepsilon_{k}} \rightarrow y^{*}$ in $L^{1}(\Omega)$. Hence, $y^{*}=\tilde{y}$ a. e. on $\Omega$. And we have that $\int_{\Omega} u_{\varepsilon_{k}}\left(y_{\varepsilon_{k}}-y^{*}\right) d x \rightarrow 0, k \rightarrow \infty$. Since $u_{\varepsilon_{k}} \rightarrow u^{*}$ in $L^{2}\left(\Omega,\left(\rho^{\varepsilon_{k}}\right)^{-1} d x\right)$ and $y_{\varepsilon_{k}} \rightarrow y^{*}$ in $H\left(\Omega, \rho^{\varepsilon_{k}} d x\right)$, and $L^{2}\left(\Omega,\left(\rho^{\varepsilon_{k}}\right)^{-1} d x\right)$ is the conjugate space to $L^{2}\left(\Omega, \rho^{\varepsilon_{k}} d x\right)$, it follows that

$$
\begin{gathered}
\int_{\Omega} f v_{\varepsilon_{k}} d x \rightarrow \int_{\Omega} f v d x, \int_{\Omega} f y_{\varepsilon_{k}} d x \rightarrow \int_{\Omega} f y^{*} d x, \\
\int_{\Omega} u_{\varepsilon_{k}} v_{\varepsilon_{k}} d x \rightarrow \int_{\Omega} u^{*} v d x, \int_{\Omega} u_{\varepsilon_{k}} y_{\varepsilon_{k}}
\end{gathered}
$$

Hence, the limit inequality for the inequality (4.15) has the form:

$$
\left\langle-\operatorname{div}\left(\rho(x) \nabla y^{*}\right), v-y^{*}\right\rangle_{H(\Omega, \rho d x)} \geq\left\langle f+u^{*}, v-y^{*}\right\rangle_{H(\Omega, \rho d x)} .
$$

Moreover, in view of previous suggestions, we have

$$
\begin{gathered}
\lim _{k \rightarrow \infty}\left\langle-\operatorname{div}\left(\rho^{\varepsilon_{k}} \nabla y_{\varepsilon_{k}}\right), v_{\varepsilon_{k}}-y_{\varepsilon_{k}}\right\rangle_{H\left(\Omega, \rho^{\varepsilon} k d x\right)} \\
=\left\langle-\operatorname{div}\left(\rho(x) \nabla y^{*}\right), v\right\rangle_{H(\Omega, \rho d x)}-\limsup _{k \rightarrow \infty} \int_{\Omega}\left(\nabla y_{\varepsilon_{k}}, \nabla y_{\varepsilon_{k}}\right)_{\mathbb{R}^{N}} \rho_{\varepsilon_{k}} d x \\
\geq\left\langle f+u^{*}, v-y^{*}\right\rangle_{H(\Omega, \rho d x)},
\end{gathered}
$$

or

$$
\begin{gathered}
\limsup _{k \rightarrow \infty} \int_{\Omega}\left(\nabla y_{\varepsilon_{k}}, \nabla y_{\varepsilon_{k}}\right)_{\mathbb{R}^{N}} \rho_{\varepsilon_{k}} d x \\
\leq\left\langle-\operatorname{div}\left(\rho(x) \nabla y^{*}\right), v\right\rangle_{H(\Omega, \rho d x)}-\left\langle f+u^{*}, v-y^{*}\right\rangle_{H(\Omega, \rho d x)}, \quad \forall v \in \tilde{K} .
\end{gathered}
$$

Having put in the last inequality $v=y^{*}$, we get

$$
\limsup _{k \rightarrow \infty} \int_{\Omega}\left|\nabla y_{\varepsilon_{k}}\right|^{2} \rho^{\varepsilon_{k}} d x \leq \int_{\Omega}\left|\nabla y^{*}\right|^{2} \rho d x,
$$

that together with the property of the lower semicontinuity with respect to the weak convergence in $L^{2}\left(\Omega, \rho^{\varepsilon_{k}} d x\right)$, gives us that $\nabla y_{\varepsilon_{k}} \rightarrow \nabla y^{*}$ in $L^{2}\left(\Omega, \rho^{\varepsilon_{k}} d x\right)^{N}$, $k \rightarrow \infty$.The proof is complete. 
As an evident consequence of this lemma and the lower semicontinuity property of the cost functional (4.6) with respect to $w$-convergence in the variable space $\mathbb{Y}\left(\Omega, \rho^{\varepsilon} d x\right)$, we have the following conclusion.

Corollary 4.1. Let $\left\{\varepsilon_{k}\right\}$ be a subsequence of indices $\{\varepsilon\}$ such that $\varepsilon_{k} \rightarrow 0$ as $k \rightarrow \infty$, and let $\left\{\left(u_{k}, y_{k}\right) \in \Xi_{\varepsilon_{k}}\right\}_{k \in \mathbb{N}}$ be a sequence of admissible solutions to corresponding perturbed problems (4.6)-(4.8) such that $\left(u_{k}, y_{k}\right)$ w-converges to $(u, y)$. Then properties (4.3) are valid.

To discuss properties (4.4)-(4.5), we give a result which is reciprocal in some sense to Lemma 4.1.

Lemma 4.2. Let $\left\{\rho^{\varepsilon}=(\rho)_{\varepsilon}\right\}_{\varepsilon>0}$ be a "direct" smoothing of a degenerate weight function $\rho(x) \geq 0$ and let $(u, y) \in \Xi_{H}$ be any admissible pair. Then there exists a relizing sequence $\left\{\left(\hat{u}_{\varepsilon}, \hat{y}_{\varepsilon}\right) \in \mathbb{Y}\left(\Omega, \rho^{\varepsilon} d x\right)\right\}_{\varepsilon>0}$ such that

$$
\begin{gathered}
\left(\hat{u}_{\varepsilon}, \hat{y}_{\varepsilon}\right) \in \Xi_{\varepsilon} \forall \varepsilon>0, \hat{u}_{\varepsilon} \rightarrow u \text { in } L^{2}\left(\Omega,\left(\rho^{\varepsilon}\right)^{-1} d x\right) ; \\
\hat{y}_{\varepsilon} \rightarrow y \text { in } L^{2}\left(\Omega, \rho^{\varepsilon} d x\right), \nabla \hat{y}_{\varepsilon} \rightarrow \nabla y \text { in } L^{2}\left(\Omega, \rho^{\varepsilon} d x\right)^{N} .
\end{gathered}
$$

Proof. Let us construct the sequence $\left\{\left(\hat{u}_{\varepsilon}, \hat{y}_{\varepsilon}\right)\right\}_{\varepsilon>0}$ as follows:

$$
\hat{u}_{\varepsilon}(x)=\int_{\mathbb{R}^{N}} Q(z) u(x+\varepsilon z) d z,
$$

$\hat{y}_{\varepsilon} \in H\left(\Omega, \rho^{\varepsilon} d x\right)$ is an $H$-solution of (4.8) corresponding to $u=\hat{u}_{\varepsilon}$.

Let us show that for every $\varepsilon>0$ the pair $\left(\hat{u}_{\varepsilon}, \hat{y}_{\varepsilon}\right)$ is admissible to the corresponding problem (4.6)-(4.8). Indeed, as follows from [10] there exists $C>0$ such that

$$
\hat{u}_{\varepsilon}(x) \leq C \int_{\Omega} u(x+\varepsilon z) d z .
$$

Taking into account the last inequality, properties of functions $\rho$ and $u$, using the replacement of variables in double integral, we have:

$$
\begin{gathered}
\left\|\hat{u}_{\varepsilon}\right\|_{L^{2}\left(\Omega, \rho^{-1} d x\right)}^{2}=\int_{\Omega}\left(\int_{\mathbb{R}^{N}} Q(z) u(x+\varepsilon z) d z\right)^{2} \rho^{-1} d x \\
\leq \int_{\Omega}\left(\int_{\Omega} u(x+\varepsilon z) d z\right)^{2} \rho^{-1} d x \leq C_{1} \int_{\Omega} \int_{\Omega} u^{2}(x+\varepsilon z) \rho^{-1} d z d x \\
=C_{2}\|u\|_{L^{2}(\Omega)}^{2}\left\|\rho^{-1}\right\|_{L^{1}(\Omega)} \leq C_{3}\|u\|_{L^{2}\left(\Omega, \rho^{-1} d x\right)}^{2}\left\|\rho^{-1}\right\|_{L^{1}(\Omega)}<\infty,
\end{gathered}
$$

where $C_{1}, C_{2}, C_{3}$ are some positive constants. Hence,

$$
\hat{u}_{\varepsilon} \in L^{2}\left(\Omega, \rho^{-1} d x\right) \subset L^{2}\left(\Omega,\left(\rho^{\varepsilon}\right)^{-1} d x\right),
$$


$\forall \varepsilon>0$. Let $T_{\varepsilon}: L^{2}(\Omega, \rho d x) \rightarrow L^{2}\left(\Omega, \rho^{\varepsilon} d x\right)$ is a "lifting" operator, constructed in (2.15). Since $\rho^{-1} u \in L^{2}(\Omega, \rho d x)$ (for details we refer to [10]), then

$$
\begin{aligned}
& \lim _{\varepsilon \rightarrow 0} \int_{\Omega} \hat{u}_{\varepsilon} \varphi\left(\rho^{\varepsilon}\right)^{-1} d x=\lim _{\varepsilon \rightarrow 0} \int_{\Omega} u(\varphi)_{\varepsilon}\left(\rho^{\varepsilon}\right)^{-1} d x \\
& \quad=\lim _{\varepsilon \rightarrow 0} \int_{\Omega} \rho^{-1} u(\varphi)_{\varepsilon}\left(\rho^{\varepsilon}\right)^{-1} \rho d x=\lim _{\varepsilon \rightarrow 0} \int_{\Omega} T_{\varepsilon}\left(\rho^{-1} u\right) \varphi\left(\rho^{\varepsilon}\right)^{-1} \rho^{\varepsilon} d x \\
& \quad=\lim _{\varepsilon \rightarrow 0} \int_{\Omega} T_{\varepsilon}\left(\rho^{-1} u\right) \varphi d x=\int_{\Omega} u \varphi \rho^{-1} d x .
\end{aligned}
$$

Taking into account properties of "lifting" operator (see Theorem 2.4), we have that $\hat{u}_{\varepsilon} \rightarrow u$ in $L^{2}\left(\Omega,\left(\rho^{\varepsilon}\right)^{-1} d x\right)$. In view of the definition of $U_{\partial}^{\varepsilon}$, we have that $\hat{u}_{\varepsilon} \in U_{\partial}^{\varepsilon}$. Thus, we conclude that the sequence $\left\{\left(\hat{u}_{\varepsilon}, \hat{y}_{\varepsilon}\right)\right\}_{\varepsilon>0} \in \Xi_{\varepsilon}$. As a result, following arguments of the proof of Lemma 4.1, we have that $\hat{y} \rightarrow y$ in $L^{2}\left(\Omega, \rho^{\varepsilon} d x\right)$ and $\nabla \hat{y}_{\varepsilon} \rightarrow \nabla y$ in $L^{2}\left(\Omega, \rho^{\varepsilon} d x\right)^{N}$ as $\varepsilon \rightarrow 0$, where $y=y(u)$, for any subsequence of $\left\{\hat{y}_{\varepsilon} \in H\left(\Omega, \rho^{\varepsilon} d x\right)\right\}_{\varepsilon>0}$ and, hence, for the entire sequence. Here $(u, y) \in \Xi_{H}$ is a given $H$-admissible solution to problem (3.3)-(3.5). This concludes the proof.

Corollary 4.2. Lemma 4.2 implies the equality $I(u, y)=\lim _{\varepsilon \rightarrow 0} I_{\varepsilon}\left(\hat{u}_{\varepsilon}, \hat{y}_{\varepsilon}\right)$.

As an obvious consequence of Definition 4.2, and Lemmas 4.1-4.2 with their Corollaries, we can give the following conclusion.

Theorem 4.3. Let $\left\{\rho^{\varepsilon}=(\rho)_{\varepsilon}\right\}_{\varepsilon>0}$ be a "direct" smoothing of a degenerate weight function $\rho(x)>0$. Then the minimization problem (3.3)-(3.5) is a weak variational limit of the sequence (4.6)-(4.8) as $\varepsilon \rightarrow 0$ with respect to the $w$-convergence in the variable space $\mathbb{Y}\left(\Omega, \rho^{\varepsilon} d x\right)$.

\section{General cinclusions}

In this paper we substantiate the validity of an $H$-attainability concept. Note that it can be considered in the case of solvability of initial degenerate optimal control problem and corresponding approximate problems. In order to verify that the set of optimal solutions to initial degenerate OCP is not empty, we invoke the concept of degenerate weight function of potential type (see for details [17]). Also for non-degenerate perturbed OCPs we construct the optimality conditions. As far as we show that at least one optimal solution to the problem (3.3)-(3.5) can be attained by optimal solutions to perturbed problems (4.6)-(4.8), and therefore, we can apply the derived optimality system for $\varepsilon>0$ small enough to characterise the attainable optimal pairs to the initial optimization problem. 


\section{References}

1. V. Chiado Piat, F. Serra Cassano, Some remarks about the density of smooth functions in weighted Sobolev spaces, J. Convex Analysis, 1 (2)(1994), 135-142.

2. C. D'Apice, U. De Maio, P. I. Kogut, Suboptimal boundary control for elliptic equations in critically perforated domains, Ann. Inst. H. Poincare' Anal. Non Lin'aire, 25 (2008), 1073-1101.

3. O.P. Kupenko, Optimal control problems in coefficients for degenerate variational inequalities of monotone type. I. Existence of optimal solutions, J. Comp. Appl. Math, 3 (106) (2011), 88-104.

4. O.P. KUPENKO, Optimal control problems in coefficients for degenerate variational inequalities of monotone type. II. Attainability problem, J. Comp. Appl. Math, 1 (107) (2012), 15-34.

5. P. Drabek, A. Kufner, F. Nicolosi, Non-Linear Elliptic Equations, Singular and Degenerate Cases, University of West Bohemia,1996

6. J. Heinonen, T. Kilpelainen, O. Martio, Nonlinear Potential Theory of Degenerate Elliptic Equations, Clarendon Press, London, 1993

7. V.I. Ivanenko, V. S. Melnik, Variational Methods in Control Problems for Distributed Systems (in Russian), Naukova Dumka, Kyiv, 1988

8. J.-L. Lions, Optimal Control of Systems Governed by Partial Differential Equations, Springer Verlag, New York, 1971

9. J.-L. Lions, Some Methods of Solving Non-Linear Boundary Value Problems, Dunod-Gauthier-Villars, Paris, 1969

10. S. E. Pastuhova, Degenerate equations of monotone type: Lavrent'ev phenomenon and attainability problems, Sbornik: Mathematics, 198 (10) (2007), 1465-1494.

11. V. V. Zhikov, A note on Sobolev spaces, J. Math. Sci (N.Y), 129 (1) (2005), 35933595 .

12. V. V. Zhikov, On Larentiev phenomenon, Russian J. Math. Phys, 3 (2) (1994), 249-269.

13. V. V. Zhikov, Weighted Sobolev spaces, Sbornik: Mathematics, 189 (8) (1998), $27-58$.

14. V.V. ZHIKov, Homogenization of elastitic problems on singular structures, Izvestija: Math, 66 (2) (2002), 299-365.

15. V. V. Zhikov, S. E. Pastuhova, Homogenization of degenerate elliptic equations, Siberian Math. Journal, 49 (1) (2006), 80-101.

16. N. V. ZADOIANCHUK, H-solvability of optimal control problem for degenerate elliptic variational inequalities, Reports of NASU, (8)(2015)

17. N. V. Zadoianchuk, O. P. Kupenko, On solvability of one class of optimal control problems for degenerate elliptic variational inequalities, J. Comp. and Appl. Math, 4 (114) (2013), 10-23. 